\title{
Near-infrared spectroscopy for detection of a significant patent ductus arteriosus
}

\author{
Valerie Y. Chock', Laura A. Rose', Jeanet V. Mante' and Rajesh Punn ${ }^{2}$
}

BACKGROUND: Near-infrared spectroscopy (NIRS) may assist with characterization of a hemodynamically significant patent ductus arteriosus (hsPDA) by measuring cerebral and renal saturation (Csat and Rsat) levels. We hypothesized that Csat and Rsat in preterm infants with an hsPDA would be decreased compared to those with no PDA or nonsignificant PDA.

METHODS: This non a-priori designed study retrospectively investigated clinical and ECHO characteristics of preterm infants <29 wk gestation who underwent routine NIRS monitoring. Logistic regression assessed association between NIRS measures and an hsPDA by ECHO.

RESULTS: Of 47 infants, 21 had a confirmed hsPDA by ECHO, 14 had a nonsignificant PDA, and 12 had no ECHO performed due to low clinical suspicion for PDA. Logistic regression adjusted for gestational age found that lower Rsat was associated with an hsPDA by ECHO (OR 0.9, 95\% Cl 0.83-0.98, $P=0.01$ ). Using ROC curves, Rsat $<66 \%$ identified an hsPDA with a sensitivity of $81 \%$ and specificity of $77 \%$, while Csat was not significant.

CONCLUSION: Low Rsat < $66 \%$ was associated with the presence of an hsPDA in the preterm infant. Csat may be preserved if cerebral autoregulation is largely intact. Bedside NIRS monitoring may reasonably increase suspicion for a significant PDA in the preterm infant.

A pproximately $70 \%$ of extremely premature infants have a hemodynamically significant patent ductus arteriosus (hsPDA) (1). A patent ductus arteriosus (PDA) maintains connection between the systemic and pulmonary circulation after birth, which can result in left-to-right shunting of blood and decreased end organ perfusion. Infants with an hsPDA are at risk for poor cerebral and renal perfusion and may be at risk for complications such as pulmonary hemorrhage, intraventricular hemorrhage, necrotizing enterocolitis, bronchopulmonary dysplasia, and death (2-5). Treatment of an hsPDA remains controversial. Historically, ibuprofen, indomethacin, or surgical ligation have been used to promote closure of the PDA, yet recent studies show a high incidence of spontaneous closure and potential inefficacy of medical or surgical treatment in reducing adverse outcomes $(6,7)$. Indomethacin or ibuprofen administration may further result in impaired renal function, intestinal perforation, altered cerebral autoregulation, or increasing hyperbilirubinemia (8-11). Complications of surgical ligation including an increased risk of bronchopulmonary dysplasia and neurodevelopmental impairment have also been described $(12,13)$. Proper characterization of the hemodynamic significance of the PDA is necessary to guide management decisions and optimize outcomes for the preterm infant.

However, no studies have found a definitive, minimally invasive technique to quantitatively assess PDA significance outside of echocardiogram (ECHO). Echocardiography can provide comprehensive hemodynamic information about the significance of a PDA, but typically requires the skills of a trained echocardiographer and evaluation by a pediatric cardiology subspecialist, which may be a barrier to timely diagnosis at some institutions. Furthermore, optimal ECHO measures to characterize the hemodynamic significance of a PDA have not yet been standardized, although several ECHO scoring systems have been proposed $(5,14)$.

Alternative real-time assessment of the hemodynamic significance of a PDA is needed. Near-infrared spectroscopy (NIRS) has been validated as a technology to noninvasively measure regional cerebral and renal oxygen saturation (Csat and Rsat) levels at the bedside $(15,16)$. Given that critical Csat measures $<40 \%$ have been associated with the occurrence of seizures, ischemic MRI lesions, and neuronal injury in neonatal cases and animal models (17-19), NIRS measures may also be useful in evaluating the significance of an infant's patent ductus arteriosus. Rsat measures are less well studied, but are notably decreased and more variable in the preterm infant compared to the term infant $(20,21)$, and may alert a clinician to hemodynamic changes earlier than Csat or blood pressure measures $(22,23)$. Determining the hemodynamic significance of a PDA based on reduced oxygenation of the brain or kidney may warrant medical or surgical closure of the ductus, as opposed to conservative management. This diagnostic capability could be of great utility to the physician.

In this observational study, we sought to determine if observed NIRS measurements were associated with the

'Division of Neonatal and Developmental Medicine, Department of Pediatrics, Stanford University School of Medicine, Palo Alto, California; ${ }^{2}$ Division of Pediatric Cardiology, Department of Pediatrics, Stanford University School of Medicine, Palo Alto, California. Correspondence: Valerie Y. Chock (vchock@stanford.edu)

Received 6 January 2016; accepted 27 May 2016; advance online publication 21 September 2016. doi:10.1038/pr.2016.148 


\section{Articles | Chocketal.}

presence or absence of an hsPDA. All preterm infants $<29 \mathrm{wk}$ gestation received NIRS monitoring as part of their routine clinical care. We compared Csat and Rsat measures of preterm infants in three categories: those with an hsPDA diagnosed by $\mathrm{ECHO}$, those whose $\mathrm{ECHOs}$ revealed a nonsignificant PDA, and those who did not receive an ECHO given lack of clinical concern for a significant PDA.

\section{RESULTS}

This consecutive sample of neonates $<29 \mathrm{wk}$ gestational age were admitted to the NICU at our institution over a 2-y period and underwent NIRS monitoring of cerebral and renal saturations as part of their routine clinical care. Thirty-five infants had both NIRS monitoring and ECHO performed, while 12 infants with low clinical suspicion for a PDA received only NIRS monitoring, for a total of 47 subjects. Twenty-two infants were not included because of lack of complete NIRS data. Of the 35 preterm infants receiving both NIRS monitoring and ECHO, 14 had a nonsignificant PDA and 21 had an hsPDA by ECHO. All infants had birth weight between $430-1,710 \mathrm{~g}$ and gestational age between 23-29 wk. Neonatal characteristics for all three groups are shown in Table 1 . There was no significant difference in perinatal variables between infants that had an ECHO done, except that more infants were treated with indomethacin or surgical ligation in the hsPDA group $(P=0.002)$. Although not statistically significant, the hsPDA group tended to have their ECHO and associated NIRS monitoring done at a later median age $(7 \mathrm{~d})$ than the nonsignificant PDA group (5 d).

The infants with low clinical suspicion for a PDA that did not have an ECHO performed were less critically ill than the rest of the cohort, with minimal use of vasopressors $(P=0.004)$, diuretics $(P=0.0005)$, or mechanical ventilation $(P=0.009)$ compared to the hsPDA group. They also did not have oliguria and more infants from this group were receiving enteral feeds. None of the infants were treated with indomethacin or surgical ligation. Neonatal differences between the "no ECHO" group and the nonsignificant PDA group did not reach statistical significance.

There was no significant difference in average Csat or Rsat measures when analyzed for 24,12 , or $6 \mathrm{~h}$ (Csat $73.2 \pm 2.2 \%$, $73.8 \pm 2.1 \%, 73.8 \pm 2.2 \%$, respectively, and Rsat $70.5 \pm 2.5 \%$, $70.9 \pm 2.4 \%, 70.5 \pm 2.8 \%$ ). Therefore, the complete 24 -h NIRS data were utilized to compare differences in NIRS measures between groups.

Table 2 depicts average NIRS measures of infants by their PDA classification group. Considering only the 35 infants that had ECHO results, those with an hsPDA had a significantly lower Rsat $(61 \pm 3 \%)$ compared to those with a nonsignificant or no PDA $(70 \pm 3 \%)(P=0.03)$. The average Csat in the hsPDA group $(68 \pm 2 \%)$ was also lower than in the nonsignificant or no PDA group ( $73 \pm 3 \%$ ), although not statistically significant $(P=0.24)$. Adding in the infants who did not have an ECHO done due to low clinical suspicion for a PDA, the hsPDA group continued to demonstrate significantly lower Rsat measures $(61 \pm 3 \%)$ compared to the group without ECHO data
$(72 \pm 2 \%)(P<0.01)$. Utilizing the entire cohort of infants, the Rsat levels were significantly lower $(P=0.003)$ and the cerebral to renal oxygenation ratios (CROR) significantly higher $(P=0.007)$ in the hsPDA group. Multivariate logistic regression with adjustment for gestational age as well as adjustment for the significant variables from Table 1 (pressor use, diuretic use, mechanical ventilation, and enteral feeds during NIRS monitoring) confirmed that lower Rsat (OR 0.9, 95\% CI 0.79$0.99, P=0.03$ ) and higher CROR (OR 150,95\% CI 1-1,000, $P$ $=0.05)$ were associated with the presence of an hsPDA.

Receiver operating characteristic (ROC) curves were generated for the entire cohort to establish cut-off values for Csat and Rsat that were associated with an hsPDA. An Rsat of $66.1 \%$ or less was associated with an hsPDA with a sensitivity and specificity of 81.0 and $76.9 \%$, respectively (Figure 1a, AUC $=0.786, P<0.0001)$. ROC curves were also generated for only those patients who had echocardiograms and yielded similar results, with a cut-off value of $66.0 \%$ and a sensitivity and specificity of 78.6 and $81.0 \%$, respectively (Figure $\mathbf{1 b}$, AUC $=0.748, P=0.0035)$. Csat, however, was not associated with the outcome variable.

\section{DISCUSSION}

A lower Rsat as measured with NIRS may indicate the presence of an hsPDA by ECHO. NIRS monitoring of the preterm infant may be helpful to further characterize the hsPDA and to guide treatment decisions. Given that Rsat measures in patients without an ECHO were significantly higher than those with a significant PDA, one could potentially use NIRS monitoring alone to increase a clinician's suspicion for an hsPDA. This bedside monitor could also provide cost-savings by directing clinicians about when to consider obtaining an ECHO. ROC curve analysis demonstrated that diminished Rsat below 66\% was associated with an hsPDA while Csat was not associated with an hsPDA. This finding held true when focusing on a subset of the population who had echocardiograms.

Preserved cerebral autoregulation likely explains the greater significance of Rsat when compared to Csat in infants with an hsPDA. Cerebral oxygenation may be maintained if autoregulatory capacity is intact, despite ongoing somatic ischemia. Similarly CROR was a significant NIRS measure in the full cohort given the overall preservation of autoregulation for the entire study group. However, several investigators have shown that cerebral autoregulation can be impaired in preterm infants (24-26). Our results also demonstrated a trend towards lower Csat levels in those infants with an hsPDA. It is possible that additional compromise of Csat levels may be indicative of impaired autoregulation in the setting of an hsPDA, and could potentially warrant earlier intervention to close the PDA.

Limited research has been published about NIRS monitoring in the context of a preterm neonate with a PDA. Studies have primarily focused on changes in cerebral oxygenation that are seen with indomethacin or ibuprofen treatment or with surgical ligation (27-34); normalization of NIRS measures has been associated with documented ductal closure. A few investigators have applied NIRS monitoring for early 
Table 1. Neonatal characteristics

\begin{tabular}{|c|c|c|c|c|}
\hline Neonatal variable & $\begin{array}{l}\text { hsPDA } \\
(n=21)\end{array}$ & $\begin{array}{l}\text { Nonsignificant PDA } \\
\qquad(n=14)\end{array}$ & $\begin{array}{l}\text { Low suspicion of PDA } \\
\quad(\text { no ECHO) }(n=12)\end{array}$ & $P$ value \\
\hline Gestational age (weeks) & $26.9(25.3-27.1)$ & $27.4(26.5-28.1)$ & $27.4(26.4-27.6)$ & 0.35 \\
\hline Male & $8(38 \%)$ & $5(36 \%)$ & $2(17 \%)$ & 0.44 \\
\hline Maternal race & & & & 0.14 \\
\hline Asian & $5(24 \%)$ & $1(7 \%)$ & $6(50 \%)$ & \\
\hline Other & $2(10 \%)$ & $0(0 \%)$ & $0(0 \%)$ & \\
\hline Age at NIRS monitoring (days) & $7(5-12)$ & $5(3-6)$ & $6(6-6)$ & 0.07 \\
\hline PDA treated with Indocin or ligation & $16(76 \%)$ & $3(21 \%)$ & $0(0 \%)$ & $<0.0001^{*}, \dagger$ \\
\hline Oliguria & $6(29 \%)$ & $1(7 \%)$ & $0(0 \%)$ & 0.07 \\
\hline Enteral feedings & $7(33 \%)$ & $8(62 \%)$ & $9(75 \%)$ & 0.05 \\
\hline
\end{tabular}

Values are median (interquartile range) or $n(\%) ;{ }^{*} P<0.01$ between $\mathrm{h}$ PPDA group and no $\mathrm{ECHO}$ group; ${ }^{\dagger} P<0.01$ between $h$ sPDA group and nonsignificant PDA group.

Table 2. NIRS measures

\begin{tabular}{|c|c|c|c|c|}
\hline NIRS measure & $\begin{array}{l}\text { hsPDA } \\
(n=21)\end{array}$ & $\begin{array}{l}\text { Nonsignificant PDA } \\
\qquad(n=14)\end{array}$ & $\begin{array}{l}\text { Low suspicion of PDA } \\
\text { (no ECHO) }(n=12)\end{array}$ & $P$ value \\
\hline Cerebral saturation (\%) & $68 \pm 2$ & $73 \pm 3$ & $68 \pm 2$ & $0.24 ; 0.6$ \\
\hline Cerebral:renal oxygenation ratio & $1.20 \pm 0.06$ & $1.06 \pm 0.04$ & $0.96 \pm 0.03$ & $0.13 ; 0.007^{*}$ \\
\hline
\end{tabular}

Values are mean \pm SEM; All $P$ values are listed first for subset of infants with echocardiographic results and then for the entire cohort with $* P<0.05$.

NIRS, near-infrared spectroscopy; PDA, patent ductus arteriosus.

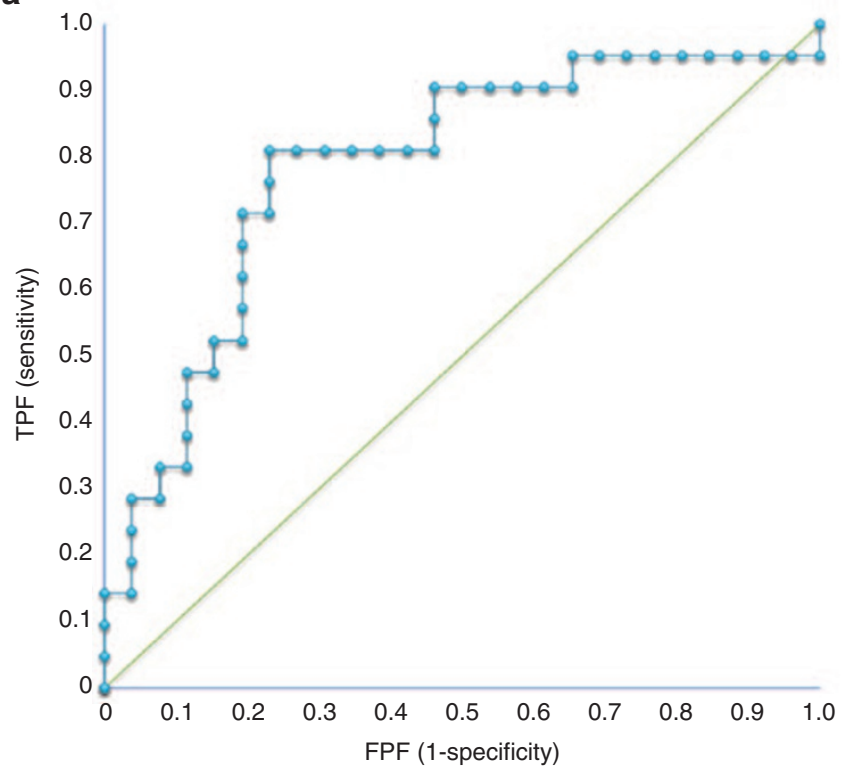

b

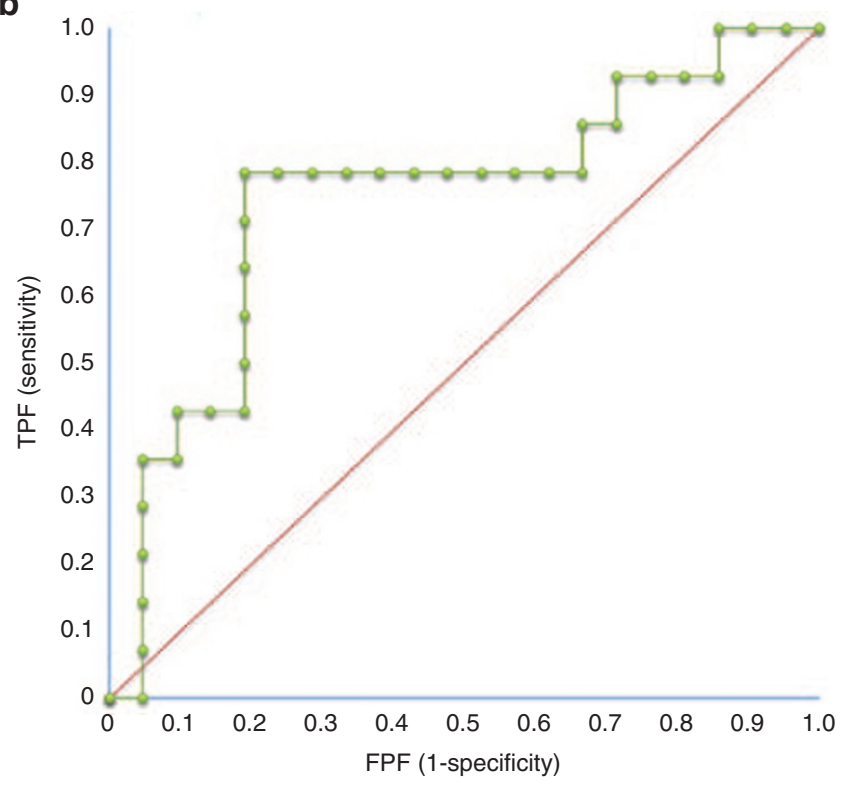

Figure 1. ROC Curves. (a) In the full cohort of infants ( $n=47$ ), renal saturation (Rsat) level $<66.1 \%$ was associated with a hemodynamically significant patent ductus arteriosus (hsPDA) with a sensitivity and specificity of 81.0 and $76.9 \%$, AUC $=0.786, P<0.0001$. (b) In the subset of infants with available echocardiographic results $(n=35)$, Rsat level $<66 \%$ was associated with an hsPDA with a sensitivity and specificity of 78.6 and $81.0 \%, A U C=0.748$, $P=0.0035$. 


\section{Articles | Chocketal.}

PDA diagnosis in the first few days of life. Lemmers et al also found a lower Csat (62 $\pm 9 \%)$ in infants with a PDA compared to matched controls without a PDA $(72 \pm 10 \%)$ by clinical or ECHO diagnosis (29). However, their study was done on preterm infants $<32 \mathrm{wk}$ gestation in the first $72 \mathrm{~h}$ of life when the ductus is more commonly open and when cerebral NIRS measures may be more unstable and more vulnerable to impaired autoregulation. Underwood et al., also studied preterm infants with NIRS monitoring in the first $4 \mathrm{~d}$ of life and similar to our study, found a decreased Rsat $<43 \%$ predicted treatment of the PDA with indomethacin or ligation (33). Given the changing approach to PDA treatment with a greater focus on conservative management while awaiting spontaneous closure and less intervention in the first few days of life, we chose to investigate the utility of NIRS monitoring at closer to a week of life when differentiation of an hsPDA is critical for directing further management. Our study uniquely demonstrates the utility of NIRS as a bedside monitor for later detection of an hsPDA.

Most similar to our study, Petrova et al. also performed NIRS measurements in preterm infants at closer to a week of life (35). These investigators found that the size of the PDA (moderate compared to large transductal diameters) did not differentiate cerebral and renal tissue oxygenation measures in preterm infants. However, the NIRS measures described in their study (68\% cerebral and 57-60\% renal) were similar to those demonstrated in our population with an hsPDA. Our study differed in that we grouped moderate and large PDA diameters together as hemodynamically significant and also included additional ECHO parameters to classify an hsPDA.

Reports of decreased mesenteric saturation in the setting of a PDA with improvement after PDA closure have also been described $(28,35)$. Our study utilized renal saturation levels rather than mesenteric saturations given the propensity for low mesenteric values with greater variability in the first few days of life (20) in preterm infants. We found renal saturation levels to be more reliably obtained with less disruption of signal integrity. However, monitoring of somatic oxygenation, whether renal or mesenteric could theoretically provide similar information about the significance of a PDA leading to a decrease in end organ oxygenation.

Limitations of this study include relatively small patient numbers at a single institution where PDA management currently favors conservative management and awaiting spontaneous ductal closure. This study was also not designed a priori, precluding a power analysis for adequate sample size. The timing of ECHO, if done at all, in the first 2 wk of life was also not standardized and could have introduced variability with age. Lack of a universal standardized ECHO scoring system for determination of an hsPDA may have misclassified infants. However, we have utilized these ECHO parameters for characterization of an hsPDA in previous research (5). Also, only 3 infants with non-significant or no PDA by ECHO had Rsat levels $<66 \%$. As all three of these infants were $<1,000 \mathrm{~g}$ and on the ventilator, their lower Rsat levels were likely influenced by low renal blood flow due to immature renal function or cardiovascular instability, and these factors should be considered as a cause of low Rsat instead of a PDA. Furthermore, interventions that could affect Csat and Rsat such as red blood cell transfusions and periods of significant hypocarbia, hypoxia, or hypotension were not excluded from the NIRS monitoring period. These factors may have affected NIRS measures beyond the effects of an hsPDA alone (36-38). It is not clear if NIRS measurements over the flank reflected oxygenation of the renal parenchyma or the surrounding tissues, as precise kidney location was not confirmed by ultrasound prior to sensor placement. However, the placement of a flank sensor below the costal margin and above the iliac crest in a neonate has previously been described in several studies as a measure of renal oxygenation $(33,35,36)$. NIRS data were averaged over a full 24 -h period to best capture overall cerebral and renal perfusion in preterm infants with the potential for a significant PDA. Lastly, the values of sensitivity and specificity in the ROC analysis were calculated on the same patient group used to obtain the threshold values, and thus represent a best-case scenario. Ideally, a future prospective study would be helpful to test an Rsat cut-off value of $66 \%$ for prediction of an hsPDA by ECHO.

\section{Conclusion}

In a population of preterm infants $<29 \mathrm{wk}$ gestation, NIRS bedside monitoring of Csat and Rsat measures was a noninvasive method to suggest the presence of an hsPDA as confirmed by ECHO. Lower Rsat levels were associated with an hsPDA, while Csat levels may have largely been preserved due to intact autoregulatory capacity. Further prospective, rigorous investigation is warranted into the utility of NIRS monitoring for detection of a significant PDA and to guide treatment decisions.

\section{METHODS \\ Subjects}

In this non-a priori designed study, a consecutive sample of eligible infants were preterm at $<29 \mathrm{wk}$ gestational age and underwent NIRS monitoring as part of their routine clinical care in the first one to $2 \mathrm{wk}$ of life. These infants were all born between September 2013 and August 2015 and admitted to the Neonatal Intensive Care Unit (NICU) at Lucile Packard Children's Hospital Stanford. Infants were not included if NIRS data were incomplete or unavailable. Infants are not treated with prophylactic indomethacin at this institution. There is currently no standardized protocol for obtaining an ECHO or for treating a suspected PDA, and such decisions were made at the discretion of the clinician. Clinicians were not blinded to NIRS measures; however, no guidelines were implemented regarding approach to NIRS measures other than to notify a clinician for Csat levels sustained at $<50 \%$. Infants who were born with congenital heart disease or other congenital or chromosomal abnormalities were excluded from this study. Expedited approval without consent was obtained from the Stanford University Human Subjects in Medical Research Committee.

\section{Data Collection}

Clinical and ECHO data if available were retrospectively obtained. Clinical variables collected included gestational age, birth weight, and PDA treatment type. Additional variables at the time of NIRS monitoring included ventilatory support, pressor and diuretic use, enteral feeding information, and presence of oliguria (defined as a urine output $<1 \mathrm{ml} / \mathrm{kg} / \mathrm{h}$ ). Infants were classified as having an hsPDA by ECHO findings of a subjectively large or very large ductal diameter, evidence of holodiastolic retrograde aortic flow, or presence of left-atrial enlargement as determined by a Pediatric Cardiologist. All other infants were 
classified as having no PDA/nonsignificant PDA by ECHO. If a subject had more than one ECHO performed in the first $2 \mathrm{wk}$ of life, the $\mathrm{ECHO}$ with the most significant findings prior to any intervention for ductal closure was utilized with its corresponding NIRS data.

NIRS monitoring of cerebral and renal saturation was standardly obtained for all preterm infants $<29 \mathrm{wk}$ gestation in the first week of life. NIRS infant sensors (INVOS 5100, Medtronic, Boulder, CO) were located on the central forehead and on either the right or left posterior flank above the iliac crest and below the costal margin (T10L2) for monitoring of Csat and Rsat levels respectively $(35,36)$. NIRS provides real time monitoring of Csat and Rsat by calculating the difference in intensity between transmitted and received near-infrared light. The near-infrared light is differentially absorbed by deoxyhemoglobin and oxyhemoglobin in the underlying tissue, allowing for calculation of a regional tissue oxygen saturation level. The CROR absolute value was calculated as an additional comparison between patients, as it has previously been demonstrated to decrease during isolated renal ischemia, but remain unchanged under normal conditions $(36,37)$. Csat, Rsat, and CROR were averaged for a 24 -h period prior to the ECHO performed to evaluate for a PDA. For the subset of infants without ECHO data, NIRS measures were analyzed for a $24-\mathrm{h}$ period beginning on day of life 6 to more closely correspond with the average age of monitoring in the group with ECHO data. Six and 12-h periods prior to ECHO were also analyzed in a random subset of 12 infants to assess for differences due to selected time period of analysis.

\section{Statistical Analyses}

Neonatal characteristics were compared between hsPDA, nonsignificant PDA, and no ECHO groups using Fisher's exact test with Freeman-Halton extension for categorical variables and KruskalWallis test for nonparametric continuous variables with Bonferroni correction for multiple comparisons. NIRS measures were normally distributed and were compared between groups with analysis of variance. The association between NIRS measures and the presence of an hsPDA was determined using multivariate logistic regression adjusted for gestational age and other determined significant variables for the entire cohort and also for the subset with confirmed ECHO results. Analyses were conducted using SAS version 9.3 (Cary, NC). ROC curves were generated to determine cut-off values for Csat or Rsat measures that were associated with an hsPDA (Analyse-it Standard version 4.10.2, Leeds, UK). Again, the entire cohort was assessed as well as only those patients with an ECHO study. The Youden index was utilized to establish cut-off values for both. Probability $(P)$ values of $<0.05$ were considered statistically significant.

\section{ACKNOWLEDGMENTS}

We thank Krisa Van Meurs, Alexis Davis, Kathi Randall, Celia Glennon, Rachael Small, Audrey Huntsberry, Keithra Ortiz, and the NeuroNICU team at Lucile Packard Children's Hospital Stanford for helping to implement NIRS monitoring practices and for archiving NIRS data during the study period. J.V.M. is also currently affiliated with Cambridge University.

\section{STATEMENT OF FINANCIAL SUPPORT}

No financial support was received for this study. We have no conflicts of interest to disclose.

\section{REFERENCES}

1. Madan JC, Kendrick D, Hagadorn JI, Frantz ID 3rd; National Institute of Child Health and Human Development Neonatal Research Network. Patent ductus arteriosus therapy: impact on neonatal and 18-month outcome. Pediatrics 2009;123:674-81.

2. Kluckow M, Evans N. Ductal shunting, high pulmonary blood flow, and pulmonary hemorrhage. J Pediatr 2000;137:68-72.

3. Evans N, Kluckow M. Early ductal shunting and intraventricular haemorrhage in ventilated preterm infants. Arch Child Fetal Neonatal Ed 1996;75:F183-6.

4. Noori S, McCoy M, Friedlich P, et al. Failure of ductus arteriosus closure is associated with increased mortality in preterm infants. Pediatrics 2009;123:e138-44.
5. Chock VY, Punn R, Oza A, et al. Predictors of bronchopulmonary dysplasia or death in premature infants with a patent ductus arteriosus. Pediatr Res 2014;75:570-5.

6. Laughon M, Bose C, Clark R. Treatment strategies to prevent or close a patent ductus arteriosus in preterm infants and outcomes. J Perinatol 2007;27:164-70.

7. Benitz WE. Treatment of persistent patent ductus arteriosus in preterm infants: time to accept the null hypothesis? J Perinatol 2010;30:241-52.

8. Watterberg KL, Gerdes JS, Cole CH, et al. Prophylaxis of early adrenal insufficiency to prevent bronchopulmonary dysplasia: a multicenter trial. Pediatrics 2004;114:1649-57.

9. Van Bel F, Van de Bor M, Stijnen T, Baan J, Ruys JH. Cerebral blood flow velocity changes in preterm infants after a single dose of indomethacin: duration of its effect. Pediatrics 1989;84:802-7.

10. Rheinlaender C, Helfenstein D, Walch E, Berns M, Obladen M, Koehne P. Total serum bilirubin levels during cyclooxygenase inhibitor treatment for patent ductus arteriosus in preterm infants. Acta Paediatr 2009;98: $36-42$.

11. Fanos V, Benini D, Verlato G, Errico G, Cuzzolin L. Efficacy and renal tolerability of ibuprofen vs. indomethacin in preterm infants with patent ductus arteriosus. Fundam Clin Pharmacol 2005;19:187-93.

12. Chorne N, Leonard C, Piecuch R, Clyman RI. Patent ductus arteriosus and its treatment as risk factors for neonatal and neurodevelopmental morbidity. Pediatrics 2007;119:1165-74.

13. Kabra NS, Schmidt B, Roberts RS, Doyle LW, Papile L, Fanaroff A; Trial of Indomethacin Prophylaxis in Preterms Investigators. Neurosensory impairment after surgical closure of patent ductus arteriosus in extremely low birth weight infants: results from the Trial of Indomethacin Prophylaxis in Preterms. J Pediatr 2007;150:229-34, 234.e1.

14. Sehgal A, McNamara PJ. Echocardiographic markers of a haemodynamically significant ductus arterious. Congenit Cardiol Today 2009;7:1-12.

15. Benni PB, Chen B, Dykes FD, et al. Validation of the CAS neonatal NIRS system by monitoring vv-ECMO patients: preliminary results. Adv Exp Med Biol 2005;566:195-201.

16. Bassan H, Gauvreau K, Newburger JW, et al. Identification of pressure passive cerebral perfusion and its mediators after infant cardiac surgery. Pediatr Res 2005;57:35-41.

17. Dent CL, Spaeth JP, Jones BV, et al. Brain magnetic resonance imaging abnormalities after the Norwood procedure using regional cerebral perfusion. J Thorac Cardiovasc Surg 2006;131:190-7.

18. Hou X, Ding H, Teng Y, et al. Research on the relationship between brain anoxia at different regional oxygen saturations and brain damage using near-infrared spectroscopy. Physiol Meas 2007;28:1251-65.

19. Kurth CD, Levy WJ, McCann J. Near-infrared spectroscopy cerebral oxygen saturation thresholds for hypoxia-ischemia in piglets. J Cereb Blood Flow Metab 2002;22:335-41.

20. McNeill S, Gatenby JC, McElroy S, Engelhardt B. Normal cerebral, renal and abdominal regional oxygen saturations using near-infrared spectroscopy in preterm infants. J Perinatol 2011;31:51-7.

21. Bernal NP, Hoffman GM, Ghanayem NS, Arca MJ. Cerebral and somatic near-infrared spectroscopy in normal newborns. J Pediatr Surg 2010;45:1306-10.

22. Hoffman GM, Ghanayem NS, Tweddell JS. Noninvasive assessment of cardiac output. Semin Thorac Cardiovasc Surg Pediatr Card Surg Annu 2005;12-21.

23. Booth EA, Dukatz C, Ausman J, Wider M. Cerebral and somatic venous oximetry in adults and infants. Surg Neurol Int 2010;1:75.

24. Wong FY, Leung TS, Austin T, et al. Impaired autoregulation in preterm infants identified by using spatially resolved spectroscopy. Pediatrics 2008;121:e604-11.

25. Soul JS, Hammer PE, Tsuji M, et al. Fluctuating pressure-passivity is common in the cerebral circulation of sick premature infants. Pediatr Res 2007;61:467-73.

26. Tsuji M, Saul JP, du Plessis A, et al. Cerebral intravascular oxygenation correlates with mean arterial pressure in critically ill premature infants. Pediatrics 2000;106:625-32. 


\section{Articles | Chocketal.}

27. Chock VY, Ramamoorthy C, Van Meurs KP. Cerebral oxygenation during different treatment strategies for a patent ductus arteriosus. Neonatology 2011;100:233-40.

28. Meier SD, Eble BK, Stapleton GE, Morales DL, Chang AC, Andropoulos DB. Mesenteric oxyhemoglobin desaturation improves with patent ductus arteriosus ligation. J Perinatol 2006;26:562-4.

29. Lemmers PM, Toet MC, van Bel F. Impact of patent ductus arteriosus and subsequent therapy with indomethacin on cerebral oxygenation in preterm infants. Pediatrics 2008;121:142-7.

30. Lemmers PM, Molenschot MC, Evens J, Toet MC, van Bel F. Is cerebral oxygen supply compromised in preterm infants undergoing surgical closure for patent ductus arteriosus? Arch Child Fetal Neonatal Ed 2010;95:F429-34.

31. Mosca F, Bray M, Lattanzio M, Fumagalli M, Tosetto C. Comparative evaluation of the effects of indomethacin and ibuprofen on cerebral perfusion and oxygenation in preterm infants with patent ductus arteriosus. J Pediatr 1997;131:549-54

32. Zaramella P, Freato F, Quaresima V, et al. Surgical closure of patent ductus arteriosus reduces the cerebral tissue oxygenation index in preterm infants: a near-infrared spectroscopy and Doppler study. Pediatr Int 2006;48:305-12.
33. Underwood MA, Milstein JM, Sherman MP. Near-infrared spectroscopy as a screening tool for patent ductus arteriosus in extremely low birth weight infants. Neonatology 2007;91:134-9.

34. Vanderhaegen J, De Smet D, Meyns B, Van De Velde M, Van Huffel S, Naulaers G. Surgical closure of the patent ductus arteriosus and its effect on the cerebral tissue oxygenation. Acta Paediatr 2008;97:1640-4.

35. Petrova A, Bhatt M, Mehta R. Regional tissue oxygenation in preterm born infants in association with echocardiographically significant patent ductus arteriosus. J Perinatol 2011;31:460-4.

36. Dani C, Pratesi S, Fontanelli G, Barp J, Bertini G. Blood transfusions increase cerebral, splanchnic, and renal oxygenation in anemic preterm infants. Transfusion 2010;50:1220-6.

37. Petrova A, Mehta R. Near-infrared spectroscopy in the detection of regional tissue oxygenation during hypoxic events in preterm infants undergoing critical care. Pediatr Crit Care Med 2006;7:449-54.

38. Vanderhaegen J, Naulaers G, Vanhole C, et al. The effect of changes in tPCO2 on the fractional tissue oxygen extraction-as measured by nearinfrared spectroscopy-in neonates during the first days of life. Eur J Paediatr Neurol 2009;13:128-34. 